\title{
Psychotherapy Training for General Psychiatrists in the Periphery of a Region
}

\author{
Peter Whewell, Consultant Psychotherapist, Dryden Road Hospital, Gateshead NE9 5BY
}

The recent substantive report Guidelines for the Training of General Psychiatrists in Psychotherapy ${ }^{1}$ makes explicit the expectations of the College in regard to psychotherapy training for trainees in general psychiatry. The report points out the particular difficulties in providing such psychotherapy training outside the teaching centres. This paper is a description of the organisation of psychotherapy training in the periphery of the Northern Region and of what may be achieved with limited resource.

The Northern Region extends from Teesside in the south to Berwick in the north, and from Sunderland in the east to Whitehaven in the west, that is an area of 10,000 square miles, and (excluding Newcastle) contains a population of some 2.5 millions. Within the region there are six training schemes for junior psychiatrists, based at Carlisle (East Cumbria), Morpeth (Gateshead and Northumberland), Sunderland (Sunderland), Middlesbrough (South Tees) and Sedgefield (South West Durham). There are approximately 60 junior psychiatrists in post at SHO and registrar level, and in addition about $20 \mathrm{GP}$ trainees at SHO level on six monthly attachments. The author was appointed single handed consultant psychotherapist in 1983 with a three session peripatetic brief for organising peripheral training in psychotherapy.

\section{Organisational principles}

These have been formulated against a background of limited training resource which have meant that only some of the recommendations in the College Guidelines can be achieved. A main aim has had to be formulated and this is the goal of practical competence in individual dynamic psychotherapy by as many trainees as possible, given considerations of aptitude, competence and adequate supervisory resource. In order to reach the position of being able to take on an individual case for dynamic psychotherapy, the trainee first needs competence in basic interviewing and secondly experience of dealing with psychotherapeutic aspects of his ordinary psychiatric caseload. Training begins therefore with a course in viewing skills, and then moves onto a seminar in which the trainee presents cases which are considered psychodynamically. Only when trainees have shown that they can relate well to patients and have gained some psychotherapeutic skill are they allowed to move on to seeing individual cases for dynamic psychotherapy under close supervision.

In addition to the main aim of practical competence in individual dynamic psychotherapy, a second aim has been to allow the trainee to gain knowledge of theoretical aspects of psychotherapy, of psychotherapeutic views on psycho- pathology, of psychotherapy research, of indications for the use of psychotherapy and the various modes and forms of psychotherapy. This knowledge is necessary for the MRCPsych Part I examination, and is accomplished by a series of lectures and seminars. A third subsidiary aim has been to try to give all the trainees an experience of group dynamics both as an aid to learning about groups and as a practical assistance to them in coping with their everyday organisational difficulties.

Even the limited aims already described need considerable input of resources, and are clearly beyond the capacity of one consultant psychotherapist. To overcome this difficulty two strategies have been employed. The first has been to centralise parts of the training to utilise resource at the centre (in this case Newcastle) whilst retaining the district as the base for the clinical practical work. The second has been to co-opt the assistance of interested and skilled consultant psychiatrists in the periphery of the region to help with the basic seminars and the individual case supervision.

In summary the basic organisation of training has been as follows:

1. At district base (a) large group seminars on the psychotherapeutic aspects of psychiatric casework; (b) small supervision groups for individual dynamic psychotherapy. 2. At central base (c) Interview skills seminars in conjunction with the academic department of psychiatry; (d) lectures and seminars on theoretical aspects of psychotherapy; (e) group courses on group process.

These sub-trainings are now discussed in more detail.

\section{Interview skills seminars}

The rationale for centralising seminars in interview skills has been to reduce splitting. Joint participation with the academic department has meant that central teaching hospital trainees and peripheral trainees have been able to join together for interview seminars which are timed for the trainees' first year in psychiatry. More importantly, each seminar has two leaders, one a member of the academic department and one a psychotherapist. This allows the trainees to develop an integrated, adaptable interviewing style that is capable of picking up both psychopathological phenomenology and psychodynamic information, with the aim of managing the interview and the patient as well as making a psychiatric and psychodynamic formulation. The format in use is that the psychiatrist and the psychotherapist present videotapes of psychiatric interviews with a particular bias towards the psychiatric and psychodynamic formulations respectively. The trainees in turn then present 
videotapes of an interview made in their base hospital and these are discussed in the seminar.

In order to reduce persecutory anxiety and to allow the group to develop into a cohesive task group, each pair of leaders takes only eight trainees and follows each group through ten seminars. Nevertheless anxiety remains at a high level until the trainee has presented his own videotape; tapes have not infrequently been forgotten or badly recorded, as evidence of the stress involved. It is apparent that the trainees in their interviews are struggling with several interlocking issues - their anxiety about the recording, managing their interaction with the patient, keeping hold of the structure of the interview, understanding the phenomenology, and integrating psychiatric and psychodynamic exploration. Two particular ways in which the patients' use of projective identification affects the interviewing process are apparent. Firstly, the over-empathic trainee interviewer is liable to be seduced away from assessment and into identification with the patient. Secondly, the more defensive trainee is likely to move into a rather hostile adherence to a rigid interview structure. It has become clear that ten interviewing seminars are not enough for this delicate and time-consuming work and that we should expand in the future to 20 or $\mathbf{3 0}$ interview sessions. Feedback from the trainees supports this view. One particular anxiety was that central trainees might out-perform or be more enthusiastic than peripheral trainees but this anxiety has proved unfounded.

2. Seminars on psychotherapeutic aspects of psychiatric casework

These seminars are run continuously at the base hospital on a weekly basis except at the outlying South Tees and East Cumbria districts which are visited once per fortnight. For the GP trainees the seminars represent their main psychotherapy training. The trainees are asked to present cases and each group develops its own format and rules. Occasionally there is group pressure to reduce anxiety and rivalry by creating a rota, in other groups the more confident bring cases first and the more timid present later. At the beginning of the life of the seminar a semi-directive technique by the leader can be used to allow confidence to develop in the predictive accuracy of a psychodynamic view; thus, for instance, a presentation may be stopped in order for the seminar group to make speculative hypotheses which may later be confirmed or disconfirmed as the material enfolds.

Dr A presented the case of a man who had been unable to work and had felt depressed since an acquaintance at his factory had been killed in an accident in which, however, the patient was not in any way involved. In a discussion about the initial material the seminar thought the depth of depression did not match the described events, and wondered whether the story was a screen for a deeper more personal trauma. One hypothesis was that a younger sibling might have died early in the patient's life. Dr A went on to confirm this as true with subsequent material.

As the seminars progress the trainees become more able to bring countertransference reactions to their patients to the seminars, and the group can help disentangle the doctor from the patient. The group itself becomes more reactive and the group's countertransference can be used by the leader.

Dr $\mathbf{G}$ brought the case of a depressed young woman, Miss $F$, whose boyfriend had left her because of her frigidity. The seminar could not get anywhere with $\mathrm{Dr} G$ in elucidating the dynamics. Eventually, with great difficulty, DrG sheepishly admitted that he had set up an Anafranil infusion on Miss F, even though she had had no specific endogenous features to her depression, because of a strong feeling that she needed it. The seminar became animated with speculations about the sexual significance of Dr G's actions. Dr $G$ said he felt sexually attacked by the group. When the seminar thought about this it seemed that the doctor-patient relationship had been re-enacted in the group, with projection of sexuality from subject ( $\mathrm{Dr} G$ ) into the object (the group) followed by violent reintrojection into $\mathrm{Dr}$ G. We wondered whether this was an avoidance of relating to Miss $F$ as a person. At follow up $\operatorname{Dr} G$ said he had been able to go back and talk to Miss $F$ about her sexual feelings who in turn had been relieved at being able to talk about her guilt about masochistic fantasies. She was able to go on and talk about feelings of being ignored and rejected by both her parents.

The trainees begin to be aware of the way in which illness can derive from breakdown of object relationship and of the difficulties in the object relationships offered within the psychiatric institution. In particular, the discontinuities of care between doctors who rotate every six months, between different professional groups, between in-patient and outpatient staff and the resulting disturbance in the patient becomes apparent. Similarly, the active splitting of staff and the pressure to re-enact rather than remember exerted by the patient becomes clear. It was to relieve the basic seminars of an increasing burden of complex organisational issues such as these that the groups on group process became an additional necessity; to protect the trainees from acting out patients' conflicts in the institution either by over-involvement or by withdrawal. One particular difficulty in these peripheral seminar groups dominated by overseas trainees has been a tendency towards dependency on the leader, with task avoidance and rivalry amongst the trainees for the attention of the leader.

\section{Individual dynamic psychotherapy}

About $60 \%$ of the psychiatric trainees have eventually progressed to taking on an individual psychotherapy case seen weekly, and under weekly supervision. In order to avoid taking on a case with whom the trainee is particularly enmeshed, the case is first assessed by the consultant psychotherapist, usually in the base hospital. Cases with more acute neurotic problems or relationship difficulties are preferred. The individual supervision groups are small, with three or four cases current for a $1 \frac{1}{2}$ hour session being the norm. Sometimes trainees not currently taking a case sit in with the group. The aim of the supervision is to allow the trainee to think about conflict, anxiety and defence and to experience and recognise the transference and its triggers in the therapy. This individual casework takes on some unique qualities in the periphery. 
The vast majority of peripheral trainees in the Northern Region have backgrounds in the Indian subcontinent, and they bring their own particular cultural bias to therapy. As a general rule they are highly perceptive and sensitive to their patient's dynamic communications, and highly accommodating to their patient's wishes.

Dr B is seeing Mr L for weekly psychotherapy following breakdown after the death of Mr L's twin. Dr B is unable to make any progress in elucidating the details of, or the affective response of the patient to, the brother's death. In one session $\mathrm{Mr} \mathrm{L}$ begins by offering physical complaints which $\mathrm{Dr} B$ is seduced into exploring in a medical way. Mr L goes on to relate a story of a friend who uses drugs in a covert, addictive way and then to talk about another friend who has problems relating to the past which he $(\mathrm{Mr}$ L) could dig up but which the friend would find painful. Mr L asks Dr B if he has heard of this drug called 'Bombay Black'. Dr B did not appreciate the obvious transference connotations of this remark.

This illustrates the sort of receptive maternal object relationship that may be offered by the Indian trainee, but which can lead to an 'addictive' therapeutic state in which the real painful issues are not confronted. The example also shows the scotoma that can develop in relation to reference to colour in the material. Similarly the peripheral trainees often have difficulty with aggressive and sexual themes.

Dr C was seeing Miss $\mathrm{P}$ for weekly psychotherapy for a problem involving an inability to separate from mother and develop an adult sexual relationship. In one session Miss $P$ brought a dream about a white van crashing into a mini. The seminar was quick to point out a transference relation between $\operatorname{Dr} C$ and the mini, Dr C being a small man, and made sexual comments which were met by embarrassment on the part of Dr C. He then revealed he had been aware of the possible aggressive sexual significance of the materia after the session but that he had modified the material presented to the seminar in order not to embarrass Dr F, a female member of the seminar.

The cultural value placed upon symbiotic mothering in Indian culture ${ }^{2}$ means that the trainees tend to feel guilty about confrontational interpretations which confirm separation between the therapist and his patient. On several occasions therapy has become stuck in a symbiotic impasse, with the trainee empathic to the self-object transference of the patient and not to the object transference. The trainee has sometimes taken the supervisor's advice to confront the patient with his transference use of the therapist, only to be left after the session with considerable countertransference disturbance.

4. Lectures on theoretical aspects of psychotherapy These lectures have been centralised into the MRCPsych course run in Newcastle for all Regional trainees. In the first year there are lectures on basic analytic concepts and on the development of psychoanalytic thought from Freud to Winnicott via Klein and Fairbairn. In the second year there are lectures on analytic views of the structure of the mind and resulting diagnostic categories, including borderline and narcissistic state, and the perversions. The third year includes seminars on psychotherapy research and assessment, and different modes of psychotherapy.

\section{Groups on group process}

In order to allow the trainees experience of group dynamics at first hand, and to allow ventilation of their complex organisational difficulties, groups on group process have been set up centrally. Eight trainees meet with two leaders weekly for an hour over two terms, with the task of discussing group process in the work setting and to study process as it occurs in the group. Because of the location it has been possible again to draw upon central resources and also to mix peripheral and central trainees.

Feedback from the leaders of these groups and from questionnaires subsequently sent to trainees indicate that these groups are worthwhile but that they cause some tension. The main tension is between members who wish to keep the group away from personal involvement and those who push towards a personal therapy group. The group process is in a compromise position here. Clearly in order to function members must present their personal involvement in the work situation and yet avoid being pulled into excessive personal revelation. The leaders are thus constantly struggling with boundary problems as well as attempting to elucidate the nature of the problems presented in the group and by the group. This all becomes active in the group's conflicts around the issue of keeping to the given task.

The leaders of the groups have reported themes concerned with power, responsibility, hierarchy, communication, scapegoating, working with other disciplines, sexual roles and culture. They also have commented upon the feelings of loneliness, isolation and helplessness experienced by trainees in their work situation. The trainees themselves almost unanimously have reported the supportive value of sharing these difficulties, as well as mentioning insight gained from understanding institutional dynamics. Groups on group process have been important in raising the morale of the peripheral trainees in particular, and have led in the past indirectly to the setting up of regular junior meetings with a task of representing the trainees' interests in hospital politics in two districts.

In the questionnaires just over $50 \%$ of the trainees said they would prefer an on-going personal group therapy. Resources within the Northern Region are not yet capable of delivering this sort of group experience, but it would seem to be an objective to be aimed for.

\section{REFERENCES}

${ }^{1}$ Royal College of Psychiatrists. Guidelines for the training of general psychiatrists in psychotherapy. (1986) Bulletin of the Royal College of Psychiatrists 10, 286-289.

${ }^{2}$ Roland, A. (1980) Psychoanalytic perspectives on personality development in India. International Journal of Psycho-Analysis, 7,73-87. 